\title{
CTRP3 as a novel biomarker in the plasma of Saudi children with autism
}

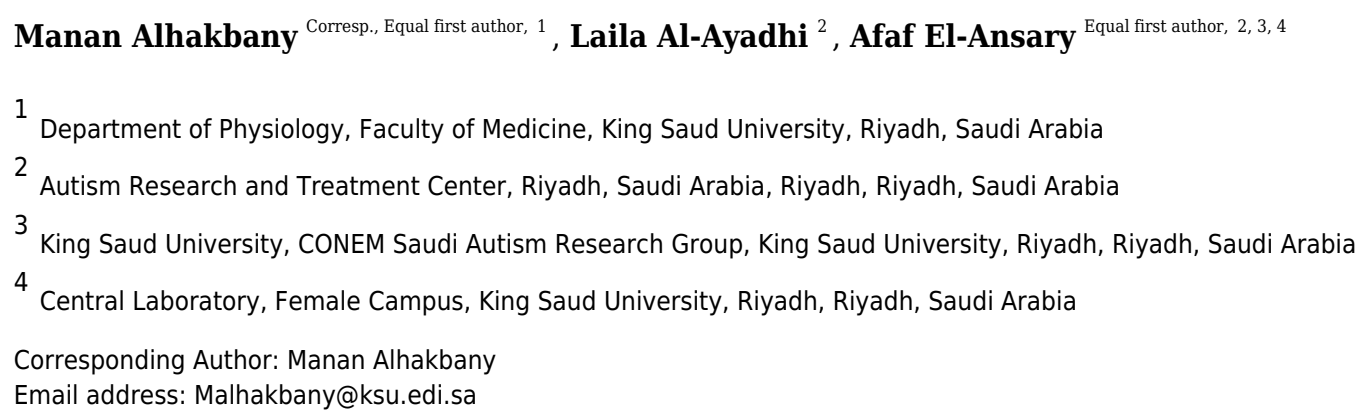

Background, Clq/tumor necrosis factor-related protein-3 (CTRP3) has diverse functions: anti-inflammation, metabolic regulation, and protection against endothelial dysfunction. Methods. The plasma level of CTRP3 in autistic patients $(n=32)$ was compared to that in controls ( $n=37)$ using ELISA. Results. CTRP3 was higher $\left(24.1 \%\right.$ with $\left.\mathrm{P}^{<} 0.05\right)$ in autistic patients than in controls. No association was observed between CTRP3 and the severity of the disorder using the Childhood Autism Rating Scale (CARS). A positive correlation between CARs and the age of patients was reported. Receiver operating characteristic (ROC) analysis demonstrated a low area under the curve (AUC) for all patients (0.636). Low AUCs were also found in the case of severe patients (0.659) compared to controls, but both values were statistically significant $(P \leq 0.05)$. Despite the small sample size, we are the first to find an association between CTRP3 and autism spectrum disorder (ASD). 
1 Title: CTRP3 as a novel biomarker in plasma of Saudi children with Autism spectrum

2 disorders

3 Authors Name:

4 Manan A. Alhakbany ${ }^{1}$

5 Laila Al-Ayadhi 1,5

6 Afaf El-Ansary $2,3,4.5$

8 Affiliation:

91 Department of Physiology, Faculty of Medicine, King Saud University, Riyadh, Saudi Arabia

102 Central Laboratory, Female Centre for Scientific and Medical Studies, King Saud University, 11 Riyadh, Saudi Arabia.

123 Therapeutic Chemistry Department, National Research Centre, Dokki, Cairo, Egypt

134 CONEM Saudi Autism Research Group, King Saud University, Riyadh, Saudi Arabia

145 Autism Research and Treatment Center, Riyadh, Saudi Arabia

*Corresponding Author:

Manan A. Alhakbany

Department of Physiology, Faculty of Medicine, King Saud University

Riyadh, Saudi Arabia

malhakbany@gmail.com 


\section{Abstract}

Background, C1q/tumor necrosis factor-related protein-3 (CTRP3) has diverse functions: anti-inflammatory, metabolic regulation, and protection against endothelial dysfunction.

Methods. The plasma level of CTRP3 in autistic patients ( $\mathrm{n}=32)$ was compared to controls $(\mathrm{n}=37)$ using ELISA.

Results. CTRP3 was higher $(24.1 \%$ with $\mathrm{P}<0.05)$ in autistic patients compared to controls. No association was observed between CTRP3 and severity of the disorder using Childhood Autism Rating Scale (CARS). A positive correlation between CARs and the age of patients was reported. Receiver operating characteristics analysis (ROC) demonstrates a low area under the curve (AUC) for the total patients (0.636). It also presents low AUCs in the case

37 of severe patients (0.659) compared to controls, but both values were statistically significant $(\mathrm{P} \leq 0.05)$.

38 Despite the small sample size, we are the first to find an association between CTRP3 and

39 autism spectrum disorder (ASD).

40 Keywords:

41 Autism spectrum disorder, biomarker, C1q/tumor necrosis factor (TNF)-related protein (CTRP3), Childhood Autism 42 Rating Scale, Receiver Operating Characteristics (ROC).

\section{Introduction}

45 Autism spectrum disorder (ASD) is a biologically neurodevelopmental disorder afflicting about one in every 59

46 children, and it is expected to increase globally. (Bjørklund et al. 2018). The increasing prevalence of ASD made it

47 a high priority for scientists and health care providers and attracted public attention (Sheldrick and Carter 2018; Xu

48 et al. 2018). Its behavior diagnoses are based on a triad of symptoms, including impairment in communication,

49 impairment in sociability, and abnormal and stereotypical behavior (Bjørklund et al., 2018). These core symptoms

50 can be detected before three years old and last for the whole lifetime (Andres 2002).

51 Recent studies mainly focus on the mechanism and the pathogenesis of ASD. Many biomarkers, which are

52 noninvasive quantitative measures, gave precise indications to specific mechanisms that can be used to provide a 
53 better understanding of the etiological mechanisms of autism and thereafter its treatment. Blood is considered a

54 potential source for detecting many diseases because it contains enormous numbers of proteins associated with the

55 physiology or pathology of diseases (Yao et al., 2021). Finding valid and predictive biomarkers for this disorder

56 will improve earlier diagnosis and intervention. Until now, no specific biomarker was found to cause autism, but

57 comparing autistic patients with peers without ASD can help to better understand of the disease.

58 Many biomarkers were related to ASD and had a significant role in its pathogenesis; immunological/inflammatory

59 markers are considered one of these biomarkers. Moreover, it was found that some ASD biomarkers were generated from lipid abnormalities (El-Ansary and Al Dera 2016).

C1q/tumor necrosis factor (TNF)-related proteins (CTRPs) family, a paralogue of adiponectin, was discovered. There are 15 members extending from CTRP1 to CTRP15. Each member comprises four different domains, an N-terminal signal domain, a short variable peptide, a collagen-like peptide, and a C-terminal globular like C1q domain (Ahima et al. 2006). Both CTRPs and adiponectin are a portion of the C1q/TNF protein, which are higher in molecular weight due to extra C1q domain proteins (Yi et al., 2012).

The CTRP family members have multiple physiological effects on metabolism, inflammation, protection against endothelial dysfunction, and angiogenesis.

CTRP3 is a novel member of this family with multiple biological functions (Peterson et al. 2010). It is detected in many tissues and organs, including the heart, liver, adipocytes, cartilage, blood vessels, monocytes, fibroblasts, colon, small intestine, pancreas, kidney, and brain (Schaffler and Buechler 2012).

71 CTRP3 is considered a strong proangiogenic and neuroprotective adipokine. CTRP3 attenuated secondary brain 72 injury after intracranial hemorrhage (ICH) in rats; it decreased brain edema, preserved the blood-brain barrier (BBB), reduced neurological deficit, and encouraged focal angiogenesis. It applies for its protective role mainly via an AMPK/HIF-1 $\alpha /$ VEGF-signaling pathway (Wang et al., 2016). CTRP3 also exerts its protective effect during

75 ICH by inhibiting oxidative stress via PKA/NADPH signaling (Yang et al., 2017).

76 Whether CTRP3, which is a member of the recently discovered adipokine family, acts as a promoter or inhibitor of 77 ASD has not been studied before. Therefore, the goal of this work was to measure the level of CTRP3 in autistic children and compare them with peers without ASD. 
79 Material and Methods

80 Study Population

81 This case-control study was conducted on 32 children diagnosed with ASD according to the 5th edition of the

82 diagnostic and statistical manual of mental disorders criteria (American Psychiatric Association 2013). Patients were 83 recruited from the Autism Research and Treatment Centre, Department of Physiology, King Saud University, Riyadh,

84 Saudi Arabia. The autistic group comprises 32 males ranging between 3 and 12 years (mean $\pm \mathrm{SD}=7.98 \pm 2.59$ years).

85 Patients who were associated with neurological disease (such as palsy and tuberous sclerosis), metabolic disorders

86 (e.g., phenylketonuria, diabetes), and autoimmune disease were excluded from the study, as metabolic disorders and

87 autoimmunity may influence the results of plasma CTRP3 levels.

88 The control group was formed of 37 age- and sex-matched healthy children; they were collected as previously described by Mustafa and Al-Ayadhi., 2015. They were not related to the children with ASD, and their ages ranged between 3 and 12 years (mean $\pm \mathrm{SD}=7.83 \pm 2.64$ years). Moreover, they had no clinical findings indicative of immunological, diabetic, chronic diseases, or neuropsychiatric disorders. The control group was the healthy older brothers of the healthy children who visit the Well Baby Clinic, King Khalid University Hospital, Faculty of Medicine, King Saud University, Riyadh, Saudi Arabia for their regular growth assessment (Mustafa and Al-Ayadhi., 2015).

This study received approval from the Ethical Committee of King Khalid University Hospital (E-10-220). The parents or the legal guardians signed informed written consent before they were included in the study.

96

97

98

\section{Study Measurements:}

\section{Clinical evaluation of autistic patients:}

The clinical evaluation depends on history taken from caregivers, clinical examination, and neuropsychiatric evaluation. Childhood Autism Rating Scale (CARS) was used to assess the severity of the disease (Mick 2005). This scale rates the child from one to four in each of fifteen areas (relating to people; emotional response; imitation; body use; object use; listening response; fear or nervousness; verbal communication; non-verbal communication; activity level; level and consistency of intellectual response; adaptation to change; visual response; taste, smell and touch response and general impressions) (Ozonoff et al., 2005). According to the scale, children who have scored 30-36 
105

106

107

108

109

110

111

112

113

114

115

116

117

118

119

120

121

122

123

124

125

126

127

128

129

have mild to moderate ASD ( $\mathrm{n}=9$ ), while those with scores ranging between 37 and 60 points have a severe degree of ASD ( $\mathrm{n}=21)$ (Schopler et al., 1988).

Blood sample collection. Ten $\mathrm{ml}$ of blood sample was collected in anticoagulant (EDTA) tubes from all the participants after overnight fasting as was previously described by Qasem et al. (2016). Plasma and RBCs were collected after centrifugation at $1000 \mathrm{rpm}$ and stored at specific temperatures till used for analysis.

Assessment of plasma CTRP3 level. Levels of trimeric CTRP3 $(<100 \mathrm{kDa})$ were measured by BioVendor human CTRP3 ELISA according to the manufacturer's protocol, with an intra-assay coefficient of variation of less than $10 \%$. Samples with CTRP3 levels below the detection limit of the assay were assigned the lowest detectable value $(0.001$ $\mu \mathrm{g} / \mathrm{ml}$ ). For accuracy, all samples were investigated as duplicate independent assays to avoid inter-assay variations and to guarantee the reproducibility of the obtained results $(\mathrm{P}>0.05)$.

Statistical analysis. A software program was used for the statistical analysis, and results were expressed as mean \pm S.D. All statistical comparisons were made by independent Student's t-test, with $\mathrm{P}$ value $<0.05$ considered significant. A Wilcoxon-Mann Whitney test is usually used when data are not normally distributed (Shapiro-Wilk's test negative). The relationship between the CTRP3, CARs, and age was calculated using the Spearman correlations test, and positive or negative correlations are listed. The receiver operating characteristics (ROC) analysis was performed as an excellent statistical tool for assessing the effectiveness of biomarkers by using the same computer software. The area under the curve (AUC) was calculated to find how a plasmatic marker can discriminate between ASD patients and healthy control participants.

\section{Results:}

\section{Demographic data:}

The demographic characteristics of children with ASD and their matched controls are shown in Table 1. Among the ASD patients, 9/30 were mild-moderate, and 21/30 were severe.

Levels of CTRP3 in total and subgroups of ASD patients compared to neurotypical healthy controls: 
130 The Shapiro test and boxplot showed that the CTRP3 data in children with ASD and healthy controls were not

131 normally distributed, with $\mathrm{P}$ values of 0.03 and 0.005 , respectively (Table 2). Tables 3 and 4 and Figure 1 demonstrate

132 the significant increase in CTRP3 in the plasma of children with ASD compared to age- and sex-matched controls.

133 ASD patients recorded $24 \%$ higher CTRP3 plasma levels than healthy controls $(\mathrm{P}<0.05)$. At the same time, there

134 was no significant difference between mild-moderate and severe autistic children $(\mathrm{P}<0.127)$, despite the $38 \%$

135 recorded increase, as shown in Table 4. Boxplots (Figure 1) show data distribution in control, total ASD, mild-

136 moderate, and severe ASD patients. CTRP3 level, the standard deviation, and the box length as a measure of data

137 dispersion of the ASD patients are considerably higher than control healthy participants and of the severe compared

138 to mild-moderate patients.

\section{Spearman correlations between CTRP3, age, and CARS:}

Table 5 and Figure 2 present the Spearman correlations among CTRP3, age, and CARS. Whereas this marker did not

142 show any independent correlations with age and CARS ( $\mathrm{P}<0.092$ and 0.750 , respectively), both variables (age and

143 CARS) were negatively correlated $(\mathrm{P}<0.044)$. The partial correlation between CTRP3 and the CARS while

144 controlling for age was insignificant, with a correlation co-officiant of $-0.210(\mathrm{p}=0.361)$.

\section{Data from ROC analysis:}

146 Table 6 demonstrates the AUCs, specificity, and sensitivity of all ASD patients and the mild-moderate and severe

147 subgroups compared to control subjects and severe autistic patients compared to mild-to-moderate participants. AUC 148 of $0.9-1.0$ shows an excellent predictive value of a biomarker, $0.8-0.9$ means a perfect marker, $0.6-0.7$ means a good 149 marker, and 0.6 means a useless marker. Based on the AUC ranges, the AUC of all patients compared to controls 150 (AUC of 0.636) and that of severe autistic patients compared to controls (AUC of 0.659) is low but within the 151 acceptable value (AUC of $0.6-0.7$ ). 
154 Discussion:

155

156 C1q TNF-related protein 3 (CTRP3) is a relatively novel hormonal factor primarily derived from adipose tissue and

157 plays a role in early childhood development (Kown et al., 2018). In the present study, the remarkably higher level of

158 CTRP3 in plasma of autistic patients compared to healthy and gender matching controls can be easily related to the disruption of BBB as a phenotypic feature in ASD. As a TNF-related protein, the increase of CTRP3 is in good agreement with multiple studies in which TNF- $\alpha$ was significantly increased in the blood and positively correlated with the severity of ASD (Xie et al., 2017).

162

In addition, Jyonouchi and collaborators (2001) found that TNF- $\alpha$ was elevated in the autistic subjects, and most of those autistic children exhibited excessive or poorly regulated innate immune responses. Moreover, Chez et al. (2007) found increased TNF- $\alpha$ in the cerebrospinal fluid of autistic children, and it was also significantly increased in the brains of autistic subjects ( $\mathrm{Li}$ et al., 2009). Although TNF- $\alpha$ was decreased in Saudi autistic patients compared to peers without ASD, this was attributed to the early increase in plasma followed by efflux to the brain through the BBB (El-

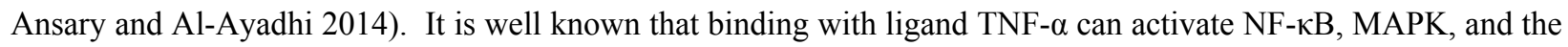
apoptosis signaling pathway (Perry et al. 2001). Based on this, the significant increase of CTRP3 in the present study can be easily related to the impaired NF- $\mathrm{KB}, \mathrm{MAPK}$, and activated apoptosis signaling pathway repeatedly recorded in autistics compared to peers without ASD (Young et al. 2011, 2012; Naik et al. 2011). A study done by Qasem et

171 al. (2018) reported a significant increase of NF-אB in plasma of Saudi patients with autism. Xu et al. (2015) suggest

172 that TNF- $\alpha$ might affect the progress of ASD through another pathway, such as the MAPK/JNK pathway, which can

173 be related to the increase of related CTRP3 reported in the present study. Gomez-Fernandez et al. (2018) showed no 174 significant difference in the level of the expression of relevant plasma cytokines, cell adhesion molecules, or growth 175 factors in children with ASD compared with peers without ASD. 
176 CTRP 3 is induced during the late stage of adipocyte differentiation and triggers the secretion of adiponectin with 177 certain regulatory metabolic functions (Yi et al., 2012). Current data proves that CTRP3 is functionally the most 178 similar homolog of adiponectin (Schaffler and Buechler 2012). Intravenously injected CTRP3 can cross the BBB and 179 increase the adiponectin levels in the cerebrospinal fluid (Wang and Scherer 2016). Recently, it was found that total 180 CTRP3 concentrations were significantly positively correlated with total cholesterol and HDL cholesterol in children

181

182 aged 7-10 years (Alamian et al., 2020). Based on this finding, the reported increased level of CTRP3 in autistic patients (Table 3 and 4) supports the association between CTRP3 and metabolic diseases. In addition, it could support the identified subgroups of individuals with ASD characterized by dyslipidemia (Luo et al., 2020; Josiane da et al., 2021). Protein kinases are essential in G-protein-coupled, receptor-mediated signal transduction and are involved in neuronal functions, gene expression, memory, and cell differentiation. The cAMP-PKA pathway is one of the most common signaling pathways (Castro et al., 2013). The activity and expression of protein kinase A (PKA), a cyclic AMPdependent protein kinase, in different areas of the postmortem brain of individuals with ASD demonstrated a significantly lower PKA level than healthy control subjects. The PKA signaling can counteract superoxide anion accumulation and prevent SOD and catalase inhibition that induced by oxidative stress in cultured astrocytes (Douiri et al. 2016). Based on the fact that Saudi autistic children are under $\mathrm{H}_{2} \mathrm{O}_{2}$ oxidative stress due to overexpression of SOD with slightly lower catalase, cAMP-PKA can be given special attention (Al-Gadani et al., 2009). PKA signaling mediates CTRP 3's anti-oxidative effects during brain injury have yet to be understood. PKA is involved in CTRP 3 mediated suppression of ROS in endothelial cells (Goldstein et al.; 2009); Yang et al. (2017) confirmed a role for PKA in the protective effects of CTRP 3's against brain injury. It is commonly known that the BBB plays an essential role in protecting the brain by limiting the influx of circulating harmful solutes, macromolecules, and cells from the blood into the brain. However, numerous studies have revealed that dysfunction of the BBB is associated with the pathogenesis of neurological disorders, including ASD, suggesting that some ASD-related proteins might be secreted from the brain into the blood as potential biomarkers. (Theoharides and Doyle, 2008; Theoharides et al., 2008; Theoharides and Zhang, 2011; Fiorentino et al., 2016).

Based on this, the remarkable increase of plasma CTRP3 in the present study can be related to BBB disruption and brain oxidative stress as confirmed etiological mechanisms in ASD. With a disrupted BBB as an autistic feature, the recorded increase of CTRP3 could be concomitant with a remarkable decrease in the brain due to efflux from the brain to blood. A study by Rai-Bhogal et al. (2018) confirmed the inhibition of PKA signaling as a pre-requisite of CTRP3 
204 protective effects with prostaglandins as a proinflammatory lipid mediator. Prostaglandins were among the elevated

205 lipid mediators previously reported by El-Ansary and Al-Ayadhi (2012) in plasma of Saudi autistic patients compared

206 to peers without ASD. In a most recent study done by Qasem et al. (2018), PGE2 and mPGES-1 were positively

207 correlated with NF- $\mathrm{BB}$ as a proinflammatory marker and associated with the dysfunction in sensory processing.

208 Table 6 demonstrates the AUCs, specificity, and sensitivity of all ASD patients compared to control subjects and

209 severe autistic patients compared to mild-to-moderate participants. The recorded AUC of all patients compared to

210 controls (AUC of 0.636) and that of severe autistic patients compared to controls (AUC of 0.659) are low but within

211 the satisfactory value known for ROC AUC (AUC of 0.6-0.7), help to accept CTRP3 as a novel predictive biomarker

212 of ASD.

213 Conclusion:

214

215 CTRP3 is a novel biomarker that had never been measured in plasma of patients with ASD; CTRP3 was higher in 216 autistic patients than controls. Therefore, it has a role in the early diagnosis of this disorder.

217 The current study's data are hindered by the relatively small sample number of participants; therefore, it needs to be replicated by a larger size of both individuals with ASD and controls. Also, all the recruited subjects in this study were males, so the conclusions cannot be anticipated in females with ASD. The inclusion of both sexes in our future studies may help to clarify the sex differences in this disorder.

Acknowledgments:

222 This project was funded by the National Plan for Science Technology and Innovation (MAARIFAH), King Abdulaziz

223 City for Science and Technology, Saudi Arabia, Award number: 08-MED 510-02. The authors want to extend their thanks to the Autism Research and Treatment Center, Riyadh, Saudi Arabia, for providing blood samples from children with autism and the control group. Special thanks to all the participants and their families for taking part in this study. 


\section{Conflict of interest}

231 The authors declare no potential conflicts of interest concerning the authorship and/or publication of this article.

\section{Ethical approval}

233 All procedures performed were following the ethical standards of the institutional and/or national research committee

234 and with the 1964 Helsinki declaration and its later amendments or comparable ethical standards.

235

236

237

238

239

240

241

242

243

244

245

246

247

248

249

250

251

252

253

254

\section{References:}

Ahima RS, Qi Y, Singhal NS, Jackson MB, Scherer PE (2006) Brain Adipocytokine Action and Metabolic Regulation. Diabetes. 55(Supplement 2): S145-S54.10.2337/db06-s018.

Alamian, A., Marrs, J. A., Clark, W. A., Thomas, K. L., \& Peterson, J. M. (2020). CTRP3 and serum triglycerides in children aged 7-10 years. PloS one, 15(12), e0241813. https://doi.org/10.1371/journal.pone.0241813.

Al-Gadani Y, El-Ansary A, Attas O, Al-Ayadhi L (2009) Metabolic biomarkers related to oxidative stress and antioxidant status in Saudi autistic children. Clin Biochem. 42(10-11):1032-40.10.1016/j.clinbiochem.2009.03.011.

American Psychiatric Association. (2013). Diagnostic and statistical manual of mental disorders (5th ed.). Arlington, VA.

Andres C (2002) Molecular genetics and animal models in autistic disorder. Brain Res Bull. 57(1):10919.10.1016/s0361-9230(01)00642-6

Bjørklund G, Meguid NA, El-Ansary A, El-Bana MA, Dadar M, Aaseth J, Hemimi M, Osredkar J, Chirumbolo S (2018) Diagnostic and Severity-Tracking Biomarkers for Autism Spectrum Disorder. J Mol Neurosci. 66(4):492511.10.1007/s12031-018-1192-1.

Castro LR, Brito M, Guiot E, Polito M, Korn CW, Hervé D, Girault JA, Paupardin-Tritsch D, Vincent P (2013) Striatal neurones have a specific ability to respond to phasic dopamine release. J Physiol. 591(13):3197214.10.1113/jphysiol.2013.252197.

Chez MG, Dowling T, Patel PB, Khanna P, Kominsky M (2007) Elevation of tumor necrosis factor-alpha in cerebrospinal fluid of autistic children. Pediatr Neurol. 36(6):361-365.10.1016/j.pediatrneurol.2007.01.012. 
255 Douiri S, Bahdoudi S, Hamdi Y, Cubì R, Basille M, Fournier A, Vaudry H, Tonon MC, Amri M, Vaudry D,

256 Masmoudi-Kouki O (2016) Involvement of endogenous antioxidant systems in the protective activity of pituitary

257 adenylate cyclase-activatin polypeptide against hydrogen peroxide-induced oxidative damages in cultured rat

258 astrocytes. J Neurochem.137 (6):913-30.10.1111/jnc.13614.

259

260

El-Ansary A, Al-Ayadhi L (2012) Lipid mediators in plasma of autism spectrum disorders. Lipids Health Dis.

261

$11: 160.10 .1186 / 1476-511 X-11-160$.

262

263

El-Ansary A, Al-Ayadhi L (2014) GABAergic/glutamatergic imbalance relative to excessive neuroinflammation in autism spectrum disorders. J Neuroinflammation. 11:189.10.1186/s12974-014-0189-0.

265

El-Ansary A, Al Dera H (2016) Biomarkers-Directed Strategies to Treat Autism. In: Wang M, Witzmann FA (ed)

Role of Biomarkers in Medicine, Intech. Open, pp 205-228

267

Fiorentino, M., Sapone, A., Senger, S., Camhi, S. S., Kadzielski, S. M., Buie, T. M., Kelly, D. L., Cascella, N., \&

Fasano, A. (2016). Blood-brain barrier and intestinal epithelial barrier alterations in autism spectrum disorders. Molecular autism, 7, 49. https://doi.org/10.1186/s13229-016-0110-z

Goldstein BJ, Scalia RG, Ma XL (2009) Protective vascular and myocardial effects of adiponectin. Nat. Clin Pract

Cardiovasc Med. 6(1): 27-35.10.1038/ncpcardio1398.

Gomez-Fernandez A, de la Torre-Aguilar MJ, Gil-Campos M, Flores-Rojas K, Cruz-Rico MD, Martin-Borreguero P,

Perez-Navero JL (2018) Children With Autism Spectrum Disorder With Regression Exhibit a Different Profile in

274 Plasma Cytokines and Adhesion Molecules Compared to Children Without Such Regression. Front Pediatr. 6:264. 0021-7557, https://doi.org/10.1016/j.jped.2020.01.003. 
281 Jyonouchi H, Sun S, Le H (2001) Proinflammatory and regulatory cytokine production associated with innate and

282 adaptive immune responses in children with autism spectrum disorders and developmental regression. J.

283 Neuroimmunol. 120(1-2):170-179.10.1016/S0165-5728(01)00421-0.

284 Kwon MR, Cress E, Clark WA, Alamian A, Lu Y, Peterson JM (2018) The adipokine C1q TNF related protein 3

285 (CTRP3) is elevated in the breast milk of obese mothers. PeerJ. 6: e4472.10.7717/peerj.4472.

286 Li, X., Chauhan, A., Sheikh, A.M., Patil, S., Chauhan, V., Li, X.M., Ji, L., Brown, T. and Malik, M., 2009. Elevated 287 immune response in the brain of autistic patients. Journal of neuroimmunology, 207(1-2), pp.111-116.

288 Luo, Y., Eran, A., Palmer, N., Avillach, P., Levy-Moonshine, A., Szolovits, P. and Kohane, I.S., 2020. A 289 multidimensional precision medicine approach identifies an autism subtype characterized by dyslipidemia. Nature 290 Medicine, 26(9), pp.1375-1379.

291 Mick KA (2005) Diagnosing autism: Comparison of the Childhood Autism Rating Scale (CARS) and the Autism 292 Diagnostic Observation Schedule (ADOS). Dissertation, Wichita State University

293

294

295

296

297

298

299

300

301

302 303
Mostafa, G. A., \& Al-Ayadhi, L. Y. (2015). Reduced levels of plasma polyunsaturated fatty acids and serum carnitine in autistic children: relation to gastrointestinal manifestations. Behavioral and brain functions: BBF, 11, 4. https://doi.org/10.1186/s12993-014-0048-2

Naik US, Gangadharan C, Abbagani K, Nagalla B, Dasari N, Manna SK (2011) A study of nuclear transcription factorkappa B in childhood autism. PLoS One. 6(5):e19488. 10.1371/journal.pone. 0019488.e19488

Ozonoff, S, Boodlin-Jones, B, \& Solomon, M. (2005). Evidence-based assessment of Autism Spectrum Disorder in children and adolescents. Journal of Clinical Child and Adolescent Psychology, 34, 523-540.

Perry RT, Collins JS, Wiener H, Acton R, Go RCP (2001) The role of TNF and its receptors in Alzheimer's disease. Neurobiol Aging. 22(6):873-883.10.1016/S0197-4580(01)00291-3.

Peterson JM, Wei Z, Wong GW (2010) C1q/TNF-related protein-3 (CTRP3), a novel adipokine that regulates hepatic glucose output. J. Biol. Chem. 285(51): 39691-39701.10.1074/jbc.M110.180695.

Peer] reviewing PDF | (2021:05:61633:2:0:NEW 14 Nov 2021) 
304 Qasem H, Al-Ayadhi L, Bjørklund G, Chirumbolo S, El-Ansary A (2018). Impaired lipid metabolism markers to 305 assess the risk of neuroinflammation in autism spectrum disorder. Metab Brain Dis 33(4):1141-1153. $306 \quad 10.1007 /$ s11011-018-0206-6

307 Qasem, H., Al-Ayadhi, L., \& El-Ansary, A. (2016). Cysteinyl leukotriene correlated with 8-isoprostane levels as 308 predictive biomarkers for sensory dysfunction in autism. Lipids in health and disease, 15, 130. 309 https://doi.org/10.1186/s12944-016-0298-0

310 Rai-Bhogal R, Wong C, Kissoondoyal A, Davidson J, Li H, Crawford DA (2018) Maternal exposure to prostaglandin 311 E (2) modifies expression of Wnt genes in mouse brain - An autism connection. Biochem Biophys Rep. 14:43$312 \quad 53.10 .1016 /$ j.bbrep.2018.03.012.

313 Schopler E, Reichler R, Renner B. The Childhood Autism Rating Scale. Los Angeles: Western Psychological Services; 3141988.

315 Schaffler A, Buechler C (2012) CTRP family: linking immunity to metabolism. Trends Endocrinol. Metab. 23(4):

316 194-204.10.1016/j.tem.2011.12.003

317 Sheldrick RC, Carter AS (2018) State-level trends in the prevalence of Autism Spectrum Disorder (ASD) from 2000 318 to 2012: A reanalysis of findings from the autism and developmental disabilities network. J. Autism Dev. Disord. 319 48(9):3086-3092.10.1007/s10803-018-3568-z.

320 Theoharides TC, Doyle R. Autism, gut-blood-brain barrier, and mast cells. J. Clin. Psychopharmacol. (2008) 28:479-

321 83. doi: 10.1097/JCP.0b013e3181845f48

322 Theoharides TC, Doyle R, Francis K, Conti P, Kalogeromitros D. Novel therapeutic targets for autism. Trends. 323 Pharmacol. Sci. (2008) 29:375-82. doi: 10.1016/j.tips.2008.06.002

324

325 Theoharides TC, Zhang B. Neuro-inflammation, blood-brain barrier, seizures and autism. $J$ 326 Neuroinflammation. (2011) 8:168. doi: 10.1186/1742-2094-8-168 
328 Wang S, Zhou Y, Yang B, Li L, Yu S, Chen Y, Zhu J, Zhao Y (2016) C1q/Tumor Necrosis Factor-Related Protein-3

329 Attenuates Brain Injury after Intracerebral Hemorrhage via AMPK-Dependent Pathway in Rat. Front Cell Neurosci.

$33010: 237.10 .3389 /$ fncel.2016.00237

331 Wang ZV, Scherer PE (2016) Adiponectin, the past two decades. J. Mol. Cell Biol. 8(2): 93-100.

$33210.1093 / \mathrm{jmcb} / \mathrm{mjw} 011$

333 Xie J, Huang L, Li X, Li H, Zhou Y, Zhu H, Pan T, Kendrick KM, Xu W (2017) Immunological cytokine profiling 334 identifies TNF- $\alpha$ as a key molecule dysregulated in autistic children. Oncotarget 8(47):82390$33582398.10 .18632 /$ oncotarget.19326

336 Xu G, Strathearn L, Liu B, Bao W (2018) Prevalence of Autism spectrum disorder among US children and adolescents, 337 2014-2016. JAMA. 319(1): 81-82.10.1001/jama.2017.17812

$338 \mathrm{Xu} \mathrm{N}, \mathrm{Li}$ X, Zhong Y (2015) Inflammatory cytokines: potential biomarkers of immunologic dysfunction in autism 339 spectrum disorders. Mediators Inflamm:531518.10.1155/2015/531518.

340 Yao Fang, Zhang Kaoyuan, Feng Chengyun, Gao Yan, Shen Liming, Liu Xukun, Ni Jiazuan. (2021) Protein

341 Biomarkers of Autism Spectrum Disorder Identified by Computational and Experimental Methods. Frontiers in 342 Psychiatry, 12, 93

343 Yang B, Wang S, Yu S, Chen Y, Li L, Zhang H, Zhao Y (2017) C1q/tumor necrosis factor-related protein 3 inhibits 344 oxidative stress during intracerebral hemorrhage via PKA signaling. Brain Res. 1657:176-184. $345 \quad 10.1016 /$ j.brainres.2016.11.016

346 Yi W, Sun Y, Yuan Y, Lau WB, Zheng Q, Wang X, Wang Y, Shang, X, Gao E, Koch WJ, Ma XL (2012) C1q/tumor 347 necrosis factor-related protein-3, a newly identified adipokine, is a novel antiapoptotic, proangiogenic, and 348 cardioprotective molecule in the ischemic mouse heart. Circulation 125(25): 3159-3169. 349 10.1161/CIRCULATIONAHA.112.099937

350 Young AM, Campbell EC, Lynch S, Suckling J, Powis SJ (2011) Aberrant NF-kappaB expression in autism spectrum 351 condition: a mechanism for neuroinflammation. Front Psychiatry. 2:27. 0.3389/fpsyt.2011.00027 
352 Young AM, Campbell EC, Lynch S, Dunn MH, Powis SJ, Suckling J (2012) Regional susceptibility to TNF- $\alpha$ 353 induction of murine brain inflammation via classical IKK/NF- $\kappa$ B signaling. PLoS one.7(6):

354 e39049.10.1371/journal.pone.0039049. e 39049.

355 Zhou, Y., Wang, J.Y., Feng, H., Wang, C., Li, L., Wu, D., Lei, H., Li, H. and Wu, L.L., 2014. Overexpression of $356 \mathrm{C} 1 \mathrm{q} /$ tumor necrosis factor-related protein-3 promotes phosphate-induced vascular smooth muscle cell calcification 357 both in vivo and in vitro. Arteriosclerosis, thrombosis, and vascular biology, 34(5), pp.1002-1010.

358

359

360

361 
Table $\mathbf{1}$ (on next page)

Demographic of children with autism and healthy control 
1

2 Table 1 : Demographic of children with autism and healthy control

3

\begin{tabular}{|c|c|c|c|}
\hline & & $\begin{array}{l}\text { Children with autism } \\
\qquad(\mathrm{n}=32)\end{array}$ & $\begin{array}{l}\text { Control group } \\
\qquad(\mathrm{n}=37)\end{array}$ \\
\hline \multirow[t]{2}{*}{ Age (in years) } & Range & $3-12$ & $3-12$ \\
\hline & Mean \pm SD & $7.98 \pm 2.59$ & $7.83 \pm 2.64$ \\
\hline Sex & Male & 32 males & 37 males \\
\hline \multirow[t]{2}{*}{ CARS scores } & Mild to moderate (30-36) & 9 & ------- \\
\hline & Severe (37-60) & 21 & ------ \\
\hline
\end{tabular}

4 
Table 2 (on next page)

Table_2_CTRP3 
2 Table 2: Test of Normality using Shapiro Test.

3

\begin{tabular}{|c|c|c|c|}
\hline Parameters & Groups & $\mathrm{N}$ & P value \\
\hline \multirow{2}{*}{$\begin{array}{c}\text { CTRP3 } \\
(\mathrm{ug} / \mathrm{ml})\end{array}$} & Control & 37 & 0.005 \\
\cline { 2 - 4 } & Patients & 32 & 0.033 \\
\hline \multirow{2}{*}{$\begin{array}{c}\text { CTRP3 } \\
(\mathrm{ug} / \mathrm{ml})\end{array}$} & Mild to Moderate & 8 & 0.041 \\
\cline { 2 - 4 } & Severe & 20 & 0.153 \\
\hline \multirow{2}{*}{$\begin{array}{c}\text { Age } \\
\text { (Years) }\end{array}$} & Mild to Moderate & 5 & 0.037 \\
\cline { 2 - 4 } & Severe & 15 & 0.269 \\
\hline
\end{tabular}

4

5 If $\mathrm{P}$ value less than or equal to 0.05 then the data is not normal distributed and If $\mathrm{P}$ value more

6 than 0.05 then the data is normal distributed

7

8 
Table 3 (on next page)

Mean \pm S.D. of CTRP3 in plasma of total autistic patients compared to control subjects. 
1

2

3 Table 3: Mean \pm S.D. of CTRP3 in plasma of total autistic patients compared to 4 control subjects.

5

\begin{tabular}{|ccccccc|}
\hline Groups & $\mathrm{N}$ & Min. & Max. & Mean \pm S.D. & $\begin{array}{c}\text { Percent } \\
\text { Change }\end{array}$ & P value \\
\hline $\begin{array}{c}\text { Control } \\
\text { (ug/ml) }\end{array}$ & 37 & 0.12 & 0.68 & $0.33 \pm 0.14$ & 100.00 & 0.050 \\
$\begin{array}{c}\text { Patients } \\
\text { (ug/ml) }\end{array}$ & 32 & 0.13 & 0.99 & $0.41 \pm 0.18$ & 124.71 & \\
\hline
\end{tabular}

6

$7 \quad *$ Comparing between groups using Mann-Whitney test

8

9

10 


\section{Table 4 (on next page)}

Mean \pm S.D. of CTRP3 in plasma of Mild to Moderate and Severe autistic patients. 
1

2

3 Table 4: Mean \pm S.D. of CTRP3 (ug/ml) in plasma of Mild to Moderate and Severe 4 autistic patients.

5

\begin{tabular}{|ccccccc|}
\hline Groups & $\mathrm{N}$ & Min. & Max. & Mean \pm S.D. & $\begin{array}{c}\text { Percent } \\
\text { Change }\end{array}$ & P value \\
\hline Mild to Moderate & 8 & 0.20 & 0.58 & $0.31 \pm 0.12$ & 100.00 & \multirow{2}{*}{0.127} \\
Severe & 20 & 0.13 & 0.99 & $0.43 \pm 0.20$ & 138.16 & \\
\hline
\end{tabular}

6

7

8

9 
Table 5 (on next page)

Spearman correlations between CTRP3, CARs, 
1 Table 5: Spearman correlations between CTRP3, CARs,

2

\begin{tabular}{|cccc|}
\hline Parameters & $\begin{array}{c}\mathrm{R} \\
\text { (Spearman } \\
\text { Correlation) }\end{array}$ & P value & \\
\hline CTRP3 with Age & -0.368 & 0.092 & $\mathrm{~N}^{\mathrm{b}}$ \\
CTRP3 with CARS & 0.061 & 0.750 & $\mathrm{P}^{\mathrm{a}}$ \\
& & & \\
Age with CARS & $-0.433^{*}$ & 0.044 & $\mathrm{~N}^{\mathrm{b}}$ \\
& & & \\
\hline
\end{tabular}

3

4 - * Correlation is significant at the 0.05 level.

5 - a Positive Correlation.

6 - ${ }^{b}$ Negative Correlation.

7 
Table 6(on next page)

ROC-Curve of CTRP3 in all patients Group according to Control Group 
Table 6: ROC-Curve of CTRP3 in all patients Group according to Control Group

3

\begin{tabular}{|l|cccccc|}
\hline \multicolumn{1}{|c|}{ CTRP3 ROC } & AUC & $\begin{array}{c}\text { Cut-off } \\
\text { value }\end{array}$ & $\begin{array}{c}\text { Sensitivity } \\
\%\end{array}$ & Specificity \% & P value & 95\% CI \\
\hline $\begin{array}{l}\text { All patients } \\
\text { according to } \\
\text { Control }\end{array}$ & 0.636 & 0.341 & $56.2 \%$ & $70.3 \%$ & 0.050 & $0.503-0.769$ \\
\hline $\begin{array}{l}\text { Mild to Moderate } \\
\text { according to } \\
\text { Control }\end{array}$ & 0.532 & 0.315 & $75.0 \%$ & $43.2 \%$ & 0.778 & $0.326-0.738$ \\
\hline $\begin{array}{l}\text { Severe according } \\
\text { to Control }\end{array}$ & 0.659 & 0.341 & $65.0 \%$ & $70.3 \%$ & 0.049 & $0.504-0.814$ \\
\hline $\begin{array}{l}\text { Severe according } \\
\text { to Mild to } \\
\text { Moderate }\end{array}$ & 0.688 & 0.372 & $60.0 \%$ & $87.5 \%$ & 0.127 & $0.479-0.896$ \\
\hline
\end{tabular}

4

5

6 
Figure 1

boxplot to show the data distribution of CTRP3 in the control and patients' groups (a), and data distribution of CTRP3 in mild to moderate and sever autistic groups (b).

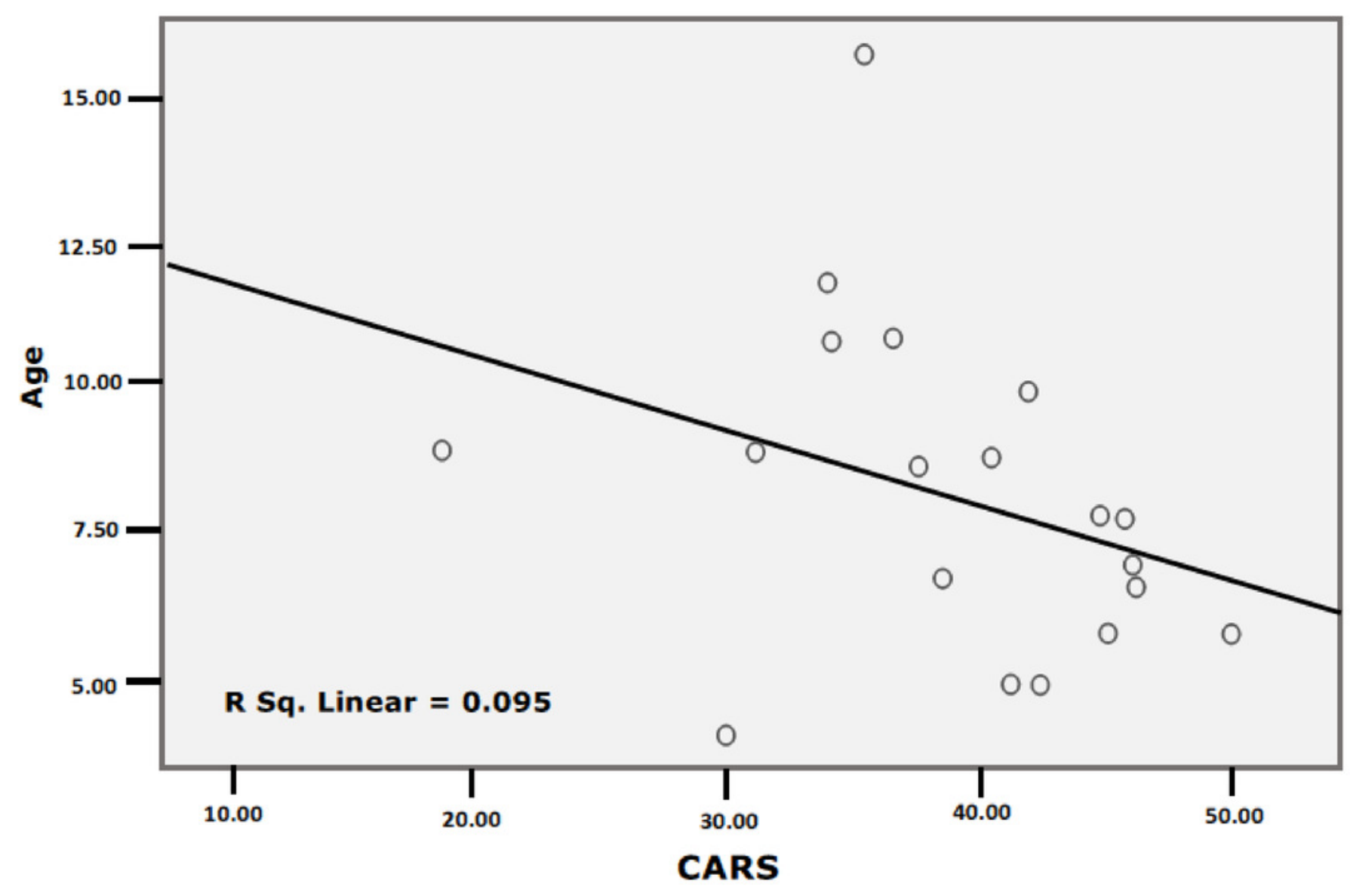


Figure 2

Correlation between CARS and Age with best fit line curve (negative correlation)
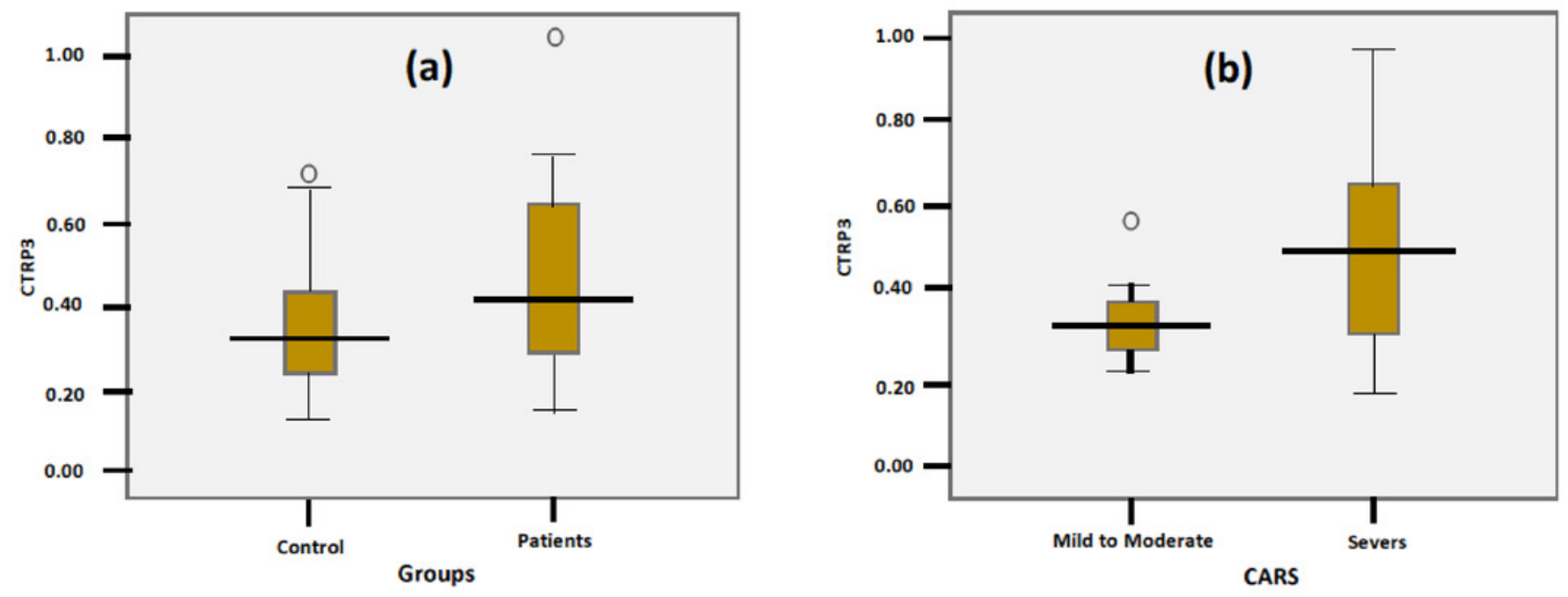